\title{
B-spline Wavelet Method for Solving Fredholm Hammerstein Integral Equation Arising from Chemical Reactor Theory
}

https://doi.org/10.1515/nleng-2017-0116

Received September 11, 2017; revised October 16, 2017; accepted December 9, 2017.

\begin{abstract}
Mathematical model for an adiabatic tubular chemical reactor which processes an irreversible exothermic chemical reaction has been considered. For steady state solution for an adiabatic tubular chemical reactor, the model can be reduced to ordinary differential equation with a parameter in the boundary conditions. Again the ordinary differential equation has been converted into a Hammerstein integral equation which can be solved numerically. B-spline wavelet method has been developed to approximate the solution of Hammerstein integral equation. This method reduces the integral equation to a system of algebraic equations. The numerical results obtained by the present method have been compared with the available results.
\end{abstract}

Keywords: B-spline, Scaling and Wavelet functions, Multiresolution analysis, Hammerstein integral equation

\section{Introduction}

In this paper, a mathematical model has been developed for an adiabatic tubular chemical reactor [1] which processes an irreversible exothermic chemical reaction. For steady state solution, the model can be reduced to ordinary differential equation with a parameter in the boundary conditions [2] as follow

$$
y^{\prime \prime}-\lambda y^{\prime}+F(\lambda, \mu, \beta, y)=0,
$$

P. K. Sahu, Department of Mathematics, School of Applied Sciences, KIIT University, Bhubaneswar, Odisha-751024, India

A. K. Ranjan, Department of Mathematics, National Institute of Technology Rourkela, Odisha-769008, India

*Corresponding Author: S. Saha Ray, Department of Mathematics, National Institute of Technology Rourkela, Odisha-769008, India, E-mail: santanusaharay@yahoo.com with boundary conditions

$$
y^{\prime}(0)=\lambda y(0), \quad y^{\prime}(1)=0,
$$

where

$$
F(\lambda, \mu, \beta, y)=\lambda \mu(\beta-y) \exp (y) .
$$

$y$ represents the steady state temperature of the reaction which has to be determined, and the parameters $\lambda, \mu$ and $\beta$ represent the Peclet number, the Damkohler number and the dimensionless adiabatic temperature rise, respectively. This problem has been studied by many researchers [1-4]. The existence of numerical solution of this problem for particular parameter range has been discussed in [14].

In order to solve the problem (1), it is converted into nonlinear Hammerstein integral equation by using Green's function. The Hammerstein integral form of eq. (1) with boundary conditions eq. (2) can be defined as

$$
y(x)=\int_{0}^{1} K(x, t) g(t, y(t)) d t, \quad 0 \leq x \leq 1,
$$

where

$$
K(x, t)= \begin{cases}e^{\lambda(x-t)}, & \text { if } 0 \leq x \leq t \\ 1, & \text { if } t \leq x \leq 1\end{cases}
$$

and

$$
g(t, y(t))=\mu(\beta-y) \exp (y) .
$$

In this paper, we consider eq. (3) as Hammerstein integral equation in the space of continuous functions on the closed interval. Throughout, we assume $\lambda$ and $\mu$ are positive, and $\beta$ is non-negative. Our main work is to solve this Hammerstein integral equation by B-spline wavelet method. Compactly supported linear semi-orthogonal Bspline wavelets have been applied to solve the integral equations of different forms [5-7]. Yousefi et al. [8] have solved age-structured population models by using the operational matrices of Bernstein polynomials. Nonlinear integral equations of the Hammerstein-type have been solved by using Double Exponential Transformation in 
[9]. Hammerstein integral equations have been solved by many authors in [10-13]. The learned researchers Saha Ray et al. have solved nonlinear Fredholm integral equations [5] and system of linear and nonlinear Fredholm integral equations $[6,7]$ by B-spline wavelet method. Also, the Bspline method has been developed for solving fractional differential equations [14]. The B-spline wavelet method converts the Hammerstein integral equation to a system of algebraic equations and that algebraic equations system again can be solved by any of the usual numerical methods. The obtained results have been compared with the results obtained by Adomian's decomposition method (ADM), contraction mapping principle (CMP) and shooting method (SM) [2].

\section{Wavelet and scaling function on bounded interval}

Let $m$ and $n$ be two positive integers and

$a=t_{-m+1}=\ldots=t_{0}<t_{1}<\ldots<t_{n}=t_{n+1}=\ldots=t_{n+m-1}=b$,

be an equally spaced knots sequence [15]. The functions

$$
\begin{gathered}
B_{m, j, T}(t)=\frac{t-t_{j}}{t_{j+m-1}-t_{j}} B_{m-1, j, T}(t)+\frac{t_{j+m}-t}{t_{j+m}-t_{j+1}} B_{m-1, j+1, T}(t), \\
j=-m+1, \ldots, n-1,
\end{gathered}
$$

and

$$
B_{1, j, T}(t)= \begin{cases}1, & \text { if } t \in\left[t_{j}, t_{j+1}\right), \\ 0, & \text { otherwise, }\end{cases}
$$

are called cardinal B-spline functions of order $m \geq 2$ for the knot sequence $T=\left\{t_{i}\right\}_{i=-m+1}^{n+m-1}$, and $\operatorname{supp} B_{m, j, T}(t)=$ $\left[t_{j}, t_{j+m}\right] \cap[a, b]$.

For the sake of simplicity, suppose $[a, b]=[0, n]$ and $t_{k}=k, \quad k=0, \ldots, n$. The $B_{m, j, T}=B_{m}(t-j), \quad j=$ $0, \ldots n-m$, are interior B-spline functions, while the remaining $B_{m, j, T}, \quad j=-m+1, \ldots,-1$ and $j=n-m, \ldots, n-1$, are boundary B-spline functions for the bounded interval $[0, n]$. Since the boundary B-spline functions at 0 are symmetric reflection of those at $n$, it is sufficient to construct only the first half functions by simply replacing $t$ with $n-t$.

By considering the interval $[a, b]=[0,1]$, at any level $j \in \mathbb{Z}^{+}$, the discretization step is $2^{-j}$, and this generates $n=2^{j}$ number of segments in $[0,1]$ with knot sequence.

$$
T^{(j)}=\left\{\begin{array}{l}
t_{-m+1}^{(j)}=\ldots=t_{0}^{(j)}, \\
t_{k}^{(j)}=\frac{k}{2^{(j)}}, \quad k=1, \ldots, n-1, \\
t_{n}^{(j)}=\ldots=t_{n+m-1}^{(j)}=1 .
\end{array}\right.
$$

Let $j_{0}$ be the level for which $2^{j_{0}} \geq 2 m-1$; for each level, $j \geq j_{0}$ the scaling function of order $m$ can be define as follows in $[6,16]$

$$
\varphi_{m, j, i}(t)=\left\{\begin{array}{cc}
B_{m, j_{0}, i}\left(2^{j-j_{0}} t\right), & i=-m+1, \ldots,-1 \\
B_{m, j_{0}, 2^{j}-m-i}\left(1-2^{j-j_{0}} t\right), & \\
i=2^{j}-m+1, \ldots, 2^{j}-1 \\
B_{m, j_{0}, 0}\left(2^{j-j_{0}} t-2^{-j_{0} i}\right), \quad i=0, \ldots, 2^{j}-m .
\end{array}\right.
$$

And the two scale relation for $m$-order semiorthogonal compactly supported B-wavelet functions are defined as follows:

$$
\psi_{m, j, i-m}=\sum_{k=i}^{2 i+2 m-2} q_{i, k} B_{m, j, k-m}, \quad i=1, \ldots, m-1,
$$

$$
\psi_{m, j, i-m}=\sum_{k=2 i-m}^{2 i+2 m-2} q_{i, k} B_{m, j, k-m}, \quad i=m, \ldots, n-m+1,
$$

$$
\psi_{m, j, i-m}=\sum_{k=2 i-m}^{n+i+m-1} q_{i, k} B_{m, j, k-m}, \quad i=n-m+2, \ldots, n,
$$

where $q_{i, k}=q_{k-2 i}$.

Hence, there are $2(m-1)$ boundary wavelets and $(n-$ $2 m+2)$ inner wavelets in the bounded interval $[a, b]$. Finally by considering the level $j$ with $j \geq j_{0}$, the B-wavelet function in $[0,1]$ can be expressed as follows:

$\psi_{m, j, i}(t)= \begin{cases}\psi_{m, j_{0}, i}\left(2^{j-j_{0}} t\right) & i=-m+1, \ldots,-1 \\ \psi_{m, 2^{j}-2 m+1-i, i}\left(1-2^{j-j_{0}} t\right) & \\ i=2^{j}-2 m+2, \ldots, 2^{j}-m & \\ \psi_{m, j_{0}, 0}\left(2^{j-j_{0}} t-2^{-j_{0} i}\right) & i=0, \ldots, 2^{j}-2 m+1 .\end{cases}$

The scaling functions $\varphi_{m, j, i}(t)$ occupy $m$ segments and the wavelet functions $\psi_{m, j, i}(t)$ occupy $2 m-1$ segments.

Therefore, the condition $2^{j} \geq 2 m-1$ must be satisfied in order to have at least one inner wavelet. In the following, the scaling functions and wavelet functions are used in this paper, for $j_{0}=j=2$ and $m=2$, are reported in $[17,18]$.

Some of the important properties relevant to the present work are given in eq. [15] as:

1) Vanishing moment: A wavelet is said to have a vanishing moment of order $m$ if

$$
\int_{-\infty}^{\infty} t^{p} \psi(t)=0 ; \quad p=0, \ldots, m-1 .
$$

All wavelets must satisfy the above condition for $p=0$. 
2)Semiorthogonality: The wavelet $\psi_{k, s}$ form a semiorthogonal basis if

$$
\left\langle\psi_{k, s}, \psi_{i, j}\right\rangle=0 ; \quad k \neq i ; \quad \forall k, s, i, j \in \mathbb{Z} .
$$

\section{Function approximation}

A function $f(x)$ defined over $[0,1]$ may be approximated by B-spline as $[19,20]$

$$
f(x)=\sum_{i=-1}^{2^{j_{0}}-1} c_{j_{0}, i} \phi_{j_{0}, i}(x)+\sum_{k=j_{0}}^{\infty} \sum_{j=-1}^{2^{k}-2} d_{k, j} \psi_{k, j}(x),
$$

where $\phi_{j_{0}, i}$ and $\psi_{k, j}$ are scaling and wavelet functions, respectively. In particular, for $j_{0}=2$, if the infinite series in eq. (14) is truncated at $M$, then eq. (14) can be written as $[16,18]$

$$
f(x) \approx \sum_{i=-1}^{2^{j_{0}}-1} c_{j_{0}, i} \phi_{j_{0}, i}(x)+\sum_{k=j_{0}}^{M} \sum_{j=-1}^{2^{k}-2} d_{k, j} \psi_{k, j}(x)=C^{T} \Psi(x),
$$

where $C$ and $\Psi$ are $\left(2^{M+1}+1\right) \times 1$ column vectors given by

$$
\begin{gathered}
C=\left[c_{2,-1}, c_{2,0}, \ldots, c_{2,3}, d_{2,-1}, \ldots, d_{2,2}, d_{3,-1}, \ldots, d_{3,6}, \ldots,\right. \\
\left.d_{M,-1}, \ldots, d_{M, 2^{M}-2}\right]^{T}, \\
\Psi=\left[\phi_{2,-1}, \phi_{2,0}, \ldots, \phi_{2,3}, \psi_{2,-1}, \ldots, \psi_{2,2}, \psi_{3,-1}, \ldots,\right. \\
\left.\psi_{3,6}, \ldots, \psi_{M,-1}, \ldots, \psi_{M, 2^{M-2}}\right]^{T},
\end{gathered}
$$

with

$$
c_{2, k}=\int_{0}^{1} f(x) \tilde{\phi}_{2, k}(x) d x, \quad k=-1,0, \ldots, 3,
$$

$d_{j, k}=\int_{0}^{1} f(x) \tilde{\psi}_{j, k}(x) d x, j=2,3 \ldots, M, k=-1,0, \ldots, 2^{j}-2$,

where $\tilde{\phi}_{2, k}(x)$ and $\tilde{\psi}_{j, k}(x)$ are dual functions of $\phi_{2, k}(x)$ and $\psi_{j, k}(x)$ respectively. These can be obtained by linear combination of $\phi_{2, k}(x), \quad k=-1,0, \ldots, 3$ and $\psi_{j, k}(x) j=$, $2,3, \ldots, M, k=-1,0, \ldots, 2^{j}-2$ as follows.

Let

$$
\Phi=\left[\phi_{2,-1}(x), \phi_{2,0}(x), \phi_{2,1}(x), \phi_{2,2}(x), \phi_{2,3}(x)\right]^{T} .
$$

$$
\bar{\Psi}=\left[\psi_{2,-1}(x), \psi_{2,0}(x), \ldots, \psi_{M, 2^{M-2}}(x)\right]^{T} .
$$

Using eqs. (7) and (20) we get,

$$
\int_{0}^{1} \Phi \Phi^{T} d x=P_{1}=\left(\begin{array}{ccccc}
\frac{1}{12} & \frac{1}{24} & 0 & 0 & 0 \\
\frac{1}{24} & \frac{1}{6} & \frac{1}{24} & 0 & 0 \\
0 & \frac{1}{24} & \frac{1}{6} & \frac{1}{24} & 0 \\
0 & 0 & \frac{1}{24} & \frac{1}{6} & \frac{1}{24} \\
0 & 0 & 0 & \frac{1}{24} & \frac{1}{12}
\end{array}\right)
$$

and from the eqs. (11) and (21) we have,

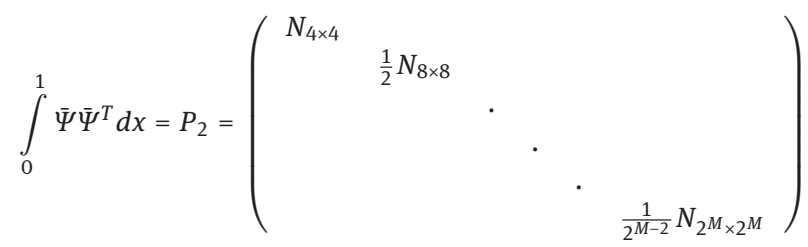

where $P_{1}$ and $P_{2}$ are $5 \times 5$ and $\left(2^{M+1}-4\right) \times\left(2^{M+1}-4\right)$ matrices, respectively, and $N$ is a five-diagonal matrix given by

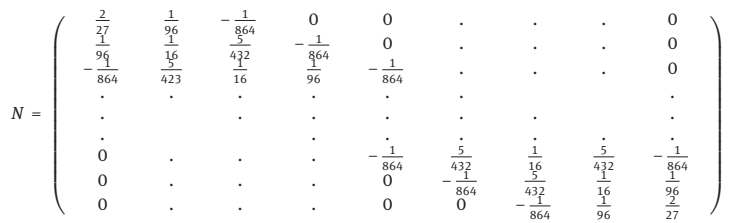

Suppose $\tilde{\Phi}$ and $\tilde{\Psi}$ are the dual functions of $\Phi$ and $\bar{\Psi}$, respectively, given by

$$
\begin{gathered}
\tilde{\Phi}=\left[\tilde{\phi}_{2,-1}(x), \tilde{\phi}_{2,0}(x), \tilde{\phi}_{2,1}(x), \tilde{\phi}_{2,2}(x), \tilde{\phi}_{2,3}(x)\right]^{T}, \\
\tilde{\Psi}=\left[\tilde{\psi}_{2,-1}(x), \tilde{\psi}_{2,0}(x), \ldots, \tilde{\psi}_{M, 2^{M}-2}(x)\right]^{T} .
\end{gathered}
$$

Combining eqs. (22) and (23), we will get

$$
\begin{aligned}
\tilde{\Psi}= & {\left[\tilde{\phi}_{2,-1}(x), \tilde{\phi}_{2,0}(x), \tilde{\phi}_{2,1}(x), \tilde{\phi}_{2,2}(x), \tilde{\phi}_{2,3}(x), \tilde{\psi}_{2,-1}(x),\right.} \\
& \left.\tilde{\psi}_{2,0}(x), \ldots, \tilde{\psi}_{M, 2^{M}-2}(x)\right]^{T} .
\end{aligned}
$$

Using eqs. (20), (22) and eqs. (21), (23) we have

$$
\int_{0}^{1} \tilde{\Phi} \Phi^{T} d x=I_{1}, \quad \int_{0}^{1} \tilde{\Psi} \bar{\Psi}^{T} d x=I_{2},
$$

where $I_{1}$ and $I_{2}$ are $5 \times 5$ and $\left(2^{(M+1)}-4\right) \times\left(2^{(M+1)}-4\right)$ identity matrices respectively. Then from matrices $P_{1}$ and $P_{2}$ we get,

$$
\tilde{\Phi}=P_{1}^{-1} \Phi, \quad \tilde{\Psi}=P_{2}^{-1} \bar{\Psi} .
$$

\section{Convergence analysis}

\section{Theorem 1:}

We assume that $f \in C^{2}[0,1]$ is represented by linear 
B-spline wavelets, where $\Psi$ has 2 vanishing moments. Then $\left|d_{j, k}\right| \leq \alpha \beta \eta^{2} \frac{2^{-3 j}}{2 !}$, where $\alpha=\max \left|f^{\prime \prime}(t)\right|_{t \in[0,1]}, \quad \beta=$ $\int_{-k}^{2^{j}-k} \tilde{\psi}(x) d x$ and $\eta \in\left(-k, 2^{j}-k\right)$.

Proof. Taylor expansion of $f \in C^{2}[0,1]$ about arbitrary $x_{0} \in[0,1]$ can be written as

$$
f(x)=f\left(x_{0}\right)+\left(x-x_{0}\right) f^{\prime}\left(x_{0}\right)+\frac{\left(x-x_{0}\right)^{2}}{2 !} f^{\prime \prime}(\xi), \quad \xi \in(0,1) .
$$

Now $f(x)$ can be presented by B-spline wavelets as

$$
f(x)=C^{T} \Psi(x)
$$

where

$$
d_{j, k}=\int_{0}^{1} f(x) \tilde{\psi}_{j, k}(x) d x .
$$

Putting eq. (27) in eq. (28), we get

$$
\begin{aligned}
d_{j, k}=\int_{0}^{1} f\left(x_{0}\right) \tilde{\psi}_{j, k}(x) d x+\int_{0}^{1}\left(x-x_{0}\right) f^{\prime}\left(x_{0}\right) \tilde{\psi}_{j, k}(x) d x \\
\quad+\int_{0}^{1} \frac{\left(x-x_{0}\right)^{2}}{2 !} f^{\prime \prime}(\xi) \tilde{\psi}_{j, k}(x) d x .
\end{aligned}
$$

Putting $x_{0}=\frac{k}{2^{j}}$ and $u=2^{j} x-k$ in eq. (29), we have

$$
\begin{gathered}
d_{j, k}=2^{-j} f\left(k / 2^{j}\right) \int_{-k}^{2^{j}-k} \tilde{\psi}_{j, k}(u) d u+2^{-2 j} f^{\prime}\left(k / 2^{j}\right) \int_{-k}^{2^{j}-k} u \tilde{\psi}_{j, k}(u) d u \\
+\frac{f^{\prime \prime}(\xi)}{2 !} 2^{-3 j} \int_{-k}^{2^{j}-k} u^{2} \tilde{\psi}_{j, k}(u) d u .
\end{gathered}
$$

Suppose $T$ is a linear transformation such that

$$
T \psi=\tilde{\psi},
$$

then taking the linear transformation $T$ of first two integral of eq. (30) we have

$$
\begin{aligned}
d_{j, k}= & 2^{-j} f\left(k / 2^{j}\right) \int_{-k}^{2^{j}-k} T\left(\psi_{j, k}(u)\right) d u \\
& +2^{-2 j} f^{\prime}\left(k / 2^{j}\right) \int_{-k}^{2^{j}-k} u T\left(\psi_{j, k}(u)\right) d u \\
& +\frac{f^{\prime \prime}(\xi)}{2 !} 2^{-3 j} \int_{-k}^{2^{j}-k} u^{2} \tilde{\psi}_{j, k}(u) d u \\
d_{j, k}= & 2^{-j} f\left(k / 2^{j}\right) T\left(\int_{-k}^{2^{j}-k} \psi_{j, k}(u) d u\right)
\end{aligned}
$$

$$
\begin{aligned}
& +2^{-2 j} f^{\prime}\left(k / 2^{j}\right) T\left(\int_{-k}^{2^{j}-k} u \psi_{j, k}(u) d u\right) \\
& +\frac{f^{\prime \prime}(\xi)}{2 !} 2^{-3 j} \int_{-k}^{2^{j}-k} u^{2} \tilde{\psi}_{j, k}(u) d u .
\end{aligned}
$$

According to vanishing moments of order $m$, i.e.

$$
\int_{-\infty}^{\infty} x^{p} \psi(x) d x=0, \quad p=0,1, \ldots, m-1,
$$

the first two integrals of eq. (31) are zero. Then we have

$$
d_{j, k}=\frac{f^{\prime \prime}(\xi)}{2 !} 2^{-3 j} \int_{-k}^{2^{j}-k} u^{2} \tilde{\psi}_{j, k}(u) d u .
$$

Applying mean value theorem for integral in eq. (32), we have

$$
d_{j, k}=\frac{f^{\prime \prime}(\xi)}{2 !} 2^{-3 j} \eta^{2} \int_{-k}^{2^{j}-k} \tilde{\psi}_{j, k}(u) d u, \quad \eta \in\left(-k, 2^{j}-k\right) .
$$

Hence

$$
\left|d_{j, k}\right| \leq \alpha \beta \eta^{2} \frac{2^{-3 j}}{2 !}
$$

\section{Theorem 2:}

Consider the previous theorem and assume that $e_{j}(x)$ be the error of approximation in $V_{j}$, then

$$
\left|e_{j}(x)\right|=O\left(2^{-2 j}\right) .
$$

Proof. Any function $f(x)=L^{2}[0,1]$ can be approximated by linear B-spline wavelets as

$$
f(x)=\sum_{k=-1}^{3} c_{k} \varphi_{2, k}+\sum_{i=2}^{\infty} \sum_{j=-1}^{2^{i}-2} d_{i, j} \psi_{i, j} .
$$

If the above function truncated at $M$, then

$$
f(x) \cong f^{\star}(x)=\sum_{k=-1}^{3} c_{k} \varphi_{2, k}+\sum_{i=2}^{M} \sum_{j=-1}^{2^{i}-2} d_{i, j} \psi_{i, j} .
$$

From eq. (33) and eq. (34), the error term can be calculated as (without loss of generality)

$$
e_{j}(x)=\sum_{l=j}^{\infty} \sum_{k=-1}^{2^{l}-2} d_{l, k} \psi_{l, k} .
$$


Setting

$$
C_{l}=\operatorname{Max}\left\{\left|\psi_{l, k}(x)\right|, \quad k=-1, \ldots, 2^{l}-2\right\} .
$$

Using Theorem 1 together with eq. (36), we obtain

$$
\left|d_{l, k} \psi_{l, k}(x)\right| \leq \alpha \beta \eta^{2} C_{l} \frac{2^{-3 l}}{2 !} .
$$

This implies

$$
\sum_{k=-1}^{2^{l}-2}\left|d_{l, k} \psi_{l, k}(x)\right| \leq \alpha \beta \eta^{2} C_{l} \frac{2^{-2 l}}{2 !} .
$$

Therefore, from eq. (35), we have

$$
\left|e_{j}(x)\right| \leq \frac{\alpha \beta}{2 !} \eta^{2} \sum_{l=j}^{\infty} C_{l} 2^{-2 l}
$$

Hence

$$
\left|e_{j}(x)\right|=O\left(2^{-2 j}\right)
$$

\section{Application of B-spline wavelet method to the Hammerstein integral equations}

In this section, we have solved the nonlinear Fredholm Hammerstein integral equation defined in eq. (3) using Bspline wavelets. First, we assume

$$
g(x, y(x))=z(x) \quad 0 \leq x \leq 1 .
$$

Now from eq. (15), we can approximate the functions $z(x)$ and $y(x)$ as

$$
\begin{aligned}
& z(x)=A^{T} \Psi(x), \\
& y(x)=B^{T} \Psi(x),
\end{aligned}
$$

where $A$ and $B$ are $\left(2^{M+1}+1\right) \times 1$ column vectors similar to $C$ as in eq. (16)

Again using the dual of wavelet functions, we can approximate $K(x, t)$ as follows.

$$
K(x, t)=\tilde{\Psi}^{T}(x) \Theta \tilde{\Psi}(x),
$$

where

$$
\Theta=\int_{0}^{1}\left[\int_{0}^{1} K(x, t) \Psi(t) d t\right] \Psi(x) d x .
$$

From eqs. (37)-(40), we will get

$$
\begin{aligned}
\int_{0}^{1} K(x, t) g(t, y(t)) d t & =\int_{0}^{1} A^{T} \Psi(t) \tilde{\Psi}^{T}(t) \Theta \tilde{\Psi}(x) d t \\
& =A^{T}\left[\int_{0}^{1} \Psi(t) \tilde{\Psi}^{T}(t) d t\right] \Theta \tilde{\Psi}(x) \\
& =A^{T} \Theta \tilde{\Psi}(x),
\end{aligned}
$$

since

$$
\int_{0}^{1} \Psi(t) \tilde{\Psi}^{T}(t) d t=I .
$$

Applying eqs. (37)-(41) in the eq. (3), we get

$$
B^{T} \Psi(x)=A^{T} \Theta \tilde{\Psi}(x) .
$$

Multiplying $\Psi^{T}(x)$ both sides of eq. (42) from the right and integrating with respect to $x$ from 0 to 1 , we get

$$
B^{T} P=A^{T} \Theta,
$$

where $P$ is a $\left(2^{M+1}+1\right) \times\left(2^{M+1}+1\right)$ square matrix given by

$$
P=\int_{0}^{1} \Psi(x) \Psi^{T}(x) d x=\left(\begin{array}{ll}
P_{1} & \\
& P_{2}
\end{array}\right),
$$

and

$$
\int_{0}^{1} \tilde{\Psi}(x) \Psi^{T}(x)=I .
$$

Eq. (43) gives a system of $\left(2^{(M+1)}+1\right)$ algebraic equations with $2\left(2^{(M+1)}+1\right)$ unknowns for $A$ and $B$ given in (38) and (39).

Again we utilize the following equation

$$
g\left(x, B^{T} \Psi(x)\right)=A^{T} \Psi(x),
$$

with the collocation points

$$
x_{s}=\frac{s-1}{2^{M+1}}, \quad s=1,2, \ldots 2^{M+1}+1 .
$$

Eq. (44) gives a system of $\left(2^{(M+1)}+1\right)$ algebraic equations with $2\left(2^{(M+1)}+1\right)$ unknowns for $A$ and $B$.

Combining eqs. (43) and (44), we have total number of $2\left(2^{(M+1)}+1\right)$ algebraic equations with same number of unknowns for $A$ and $B$. Solving the system for the unknown coefficients in the vectors $A$ and $B$, we can obtain the solution $y(x)=B^{T} \Psi(x)$. The numerical results obtained by B-spline wavelet method are cited in Table 1. Also the Table 1 cites the comparison of results obtained by 

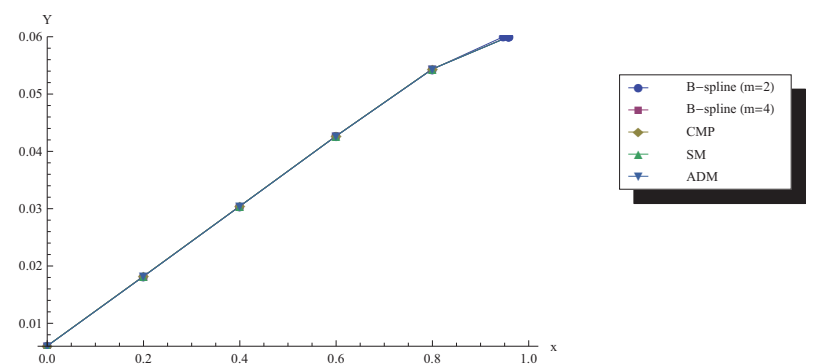

Fig. 1: Numerical results obtained by B-spline wavelet method with the results of other available methods in ref. [2] (for $\lambda=10, \beta=3$ and $\mu=0.02$ )

present method and the methods given in ref. [2] (i.e. Adomian's decomposition method, Contraction mapping principle and Shooting method). Since, the exact solution of this problem is not known, from the figure it manifests that we obtained accurate results by B-spline wavelet method in compare to other methods. As, the error of approximation by B-spline wavelet method is $O\left(2^{-2 j}\right)$, we will get more accurate result by increasing the value of $j$ (the level of resolution). Also, from the table, it is clear that the results obtained by B-spline method are more accurate than the results obtained by other method.

Table 1: Comparison of numerical results obtained by B-spline wavelet method with the results of other available methods in ref. [2] (for $\lambda=10, \beta=3$ and $\mu=0.02$ )

\begin{tabular}{llclll}
\hline $\boldsymbol{x}$ & $\begin{array}{c}\text { B-spline wavelet method } \\
\boldsymbol{M = \mathbf { 2 }}\end{array}$ & $\boldsymbol{M}=\mathbf{4}$ & $\begin{array}{l}\text { CMP } \\
{[2]}\end{array}$ & $\begin{array}{l}\text { SM } \\
{[2]}\end{array}$ & $\begin{array}{l}\text { ADM } \\
{[2]}\end{array}$ \\
\hline 0.0 & 0.006045 & 0.006048 & 0.006079 & 0.006048 & 0.006048 \\
0.2 & 0.018194 & 0.018193 & 0.018224 & 0.018192 & 0.018192 \\
0.4 & 0.030424 & 0.030424 & 0.030456 & 0.030424 & 0.030424 \\
0.6 & 0.042675 & 0.042669 & 0.042701 & 0.042669 & 0.042669 \\
0.8 & 0.054332 & 0.054368 & 0.054401 & 0.054371 & 0.054371 \\
1.0 & 0.062030 & 0.061505 & 0.061459 & 0.061458 & 0.061458 \\
\hline
\end{tabular}

\section{Conclusion}

In this paper, the compactly supported semiorthogonal linear B-Spline Wavelets have been applied to solve the nonlinear Hammerstein integral equation. We have solved a model for an adiabatic tubular chemical reactor theory which forms a nonlinear Hammerstein integral equation. Using this method, the integral equation has been reduced to a system of algebraic equations. The numerical results obtained by present method have been compared with the results obtained by Contraction mapping principle, Shooting method, and Adomian's decomposition method and this comparison justifies that the present method gives accurate results with regard to other methods if we increase the value of $M$.

Acknowledgement: The authors would like to express their sincere thanks and gratitude to the anonymous learned reviewers for their kind suggestions for the betterment and improvement of the present paper.

\section{References}

[1] Poore AB. A tubular chemical reactor model. A Collection of Nonlinear Model Problems Contributed to the Proceedings of the AMS-SIAM, 1989, 28-31.

[2] Madbouly NM, McGhee DF, Roach GF. Adomian's method for Hammerstein integral equations arising from chemical reactor theory. Applied Mathematics and Computation, 2001, 117, 241-249.

[3] Heinemann R, Poore A. Multiplicity stability and oscillatory dynamics of the tubular reactor. Chemical Engineering Science, 1981, 36, 1411-1419.

[4] Heinemann R, Poore A. The effect of activation energy on tubular reactor multiplicity. Chemical Engineering Science, 1982, 37, 128-131.

[5] Sahu PK, Saha Ray S. Numerical Approximate Solutions of Nonlinear Fredholm Integral Equations of Second Kind Using B-spline Wavelets and Variational Iteration Method. CMES, 2013, 93(2), 91-112.

[6] Sahu PK, Saha Ray S. Numerical solutions for the system of Fredholm integral equations of second kind by a new approach involving semiorthogonal B-spline wavelet collocation method. Applied Mathematics and Computation, 2014, 234, 368-379.

[7] Sahu PK, Saha Ray S. A new approach based on semiorthogonal B-spline wavelets for the numerical solutions of the system of nonlinear Fredholm integral equations of second kind. Computational and Applied Mathematics, 2014, 33(3), 859-872.

[8] Yousefi SA, Behroozifar M, Dehghan M. Numerical solution of the nonlinear age-structured population models by using the operational matrices of Bernstein polynomials. Applied Mathematical Modelling, 2012, 36, 945-963.

[9] Zarebnia M. Solving Nonlinear Integral Equations of the Hammerstein-type by Using Double Exponential Transformation. Australian Journal of Basic and Applied Sciences, 2010, 4(8), 3433-3440.

[10] Maleknejad K, Hashemizadeh E, Basirat B. Computational method based on Bernstein operational matrices for nonlinear Volterra-Fredholm-Hammerstein integral equations. Commun. Nonlinear Sci. Numer. Simulat., 2012, 17, 52-61.

[11] Lakestani M, Razzaghi M, Dehghan M. Solution of nonlinear Fredholm-Hammerstein integral equations by using semiorthogonal Spline wavelets. Mathematical Problems in Engineering, 2005, 2005(1), 113-121.

[12] Mahmoudi Y. Wavelet Galerkin method for numerical solution of nonlinear integral equation. Applied Mathematics and Com- 
putation, 2005, 167, 1119-1129.

[13] Rashidinia J, Zarebnia M. New approach for numerical solution of Hammerstein integral equations. Applied Mathematics and Computation, 2007, 185, 147-154.

[14] Jafari H, Tajadodi H, Baleanu D. A Numerical Approach for Fractional Order Riccati Differential Equation Using B-Spline Operational Matrix. Fractional Calculus and Applied Analysis, 2015, 18(2), 387-399.

[15] Mallat SG. A Wavelet Tour of Signal Processing. Academic Press, 1999.

[16] Maleknejad K, Sahlan MN. The method of moments for solution of second kind Fredholm integral equations based on B-spline wavelets. Int. J. Comp. Math., 2010, 87(7), 1602-1616.
[17] Goswami JC, Chan AK, Chui CK. On solving First-kind integral equations using wavelets on a bounded interval. IEEE Trans Antennas Propag., 1995, 43, 614-622.

[18] Lakestani M, Razzaghi M, Dehghan M. Semiorthogonal Spline Wavelet Approximation for Fredholm Integro-Differential Equation. Mathematical Problem in Engineering, 2006, 2006, 96184, 1-12.

[19] Chui CK. An Introduction to Wavelets. vol 1. Academic press limited, Massachusetts, 1992.

[20] Goswami JC, Chan AK. Fundamentals of wavelets: theory, algorithms and applications. Wiley, New York, 2011. 